\title{
Livelihood Assistance System for Vulnerable Road Users in Suburban Residential Areas based on Mutual Assis- tance
}

\author{
Shimpei Matsumoto ${ }^{*}$, Nobuyuki Ohigashi ${ }^{\dagger}$, \\ Takashi Hasuike
}

\begin{abstract}
Hiroshima City is surrounded by mountains on its three sides, the east, north, and west, except the south facing the sea, and besides, its urban area places delta region. The flatland of the urban area in Hiroshima City is limited, so its land prices are exceedingly higher than other areas. Due to these conditions, most of the citizens live in residential estates at the slope of these mountains surrounding the city. However, the living environment of the suburban slope residential estates doesn't seem so suitable for elderly people who are difficult to routinely go out, usually called as "vulnerable road users". Our previous study showed the concept of information sharing system to support the transportation of residents living in a place where public transportation is not sufficiently developed. In this paper, based on the investigation results on resident's traffic attitude in the slope residential estates at Itsukaichi District, Hiroshima City, and also our previous works, we design and develop a web service to support vulnerable road user's daily life in suburban slope residential estates. The role of the proposed system is to facilitate the encounter between local community people, to provide the opportunity of resource sharing, to generate some kinds of transportation means, and to support the difficulties in daily life, and eventually, the proposed system tries to solve the issues of vulnerable road users.
\end{abstract}

Keywords: livelihood assistance, vulnerable road users, suburban residential area, web system, sharing economy.

\section{Introduction}

Hiroshima City is surrounded by mountains on its three sides, the east, north, and west, except the south facing the sea, and besides, its urban area places delta region. The area of the urban area in Hiroshima City is limited, so the land prices near the urban area are exceedingly higher than a common urban area in Japan. Due to these conditions, the majority of the population is concentrated in the slope residential estates at the surrounding mountains

* Hiroshima Institute of Technology, Hiroshima, Japan

$\dagger$ Hiroshima Institute of Technology, Hiroshima, Japan

* Waseda University, Tokyo, Japan 
of the city. However, since the residential land development of these suburban residential areas was completed some years ago, the commercial function has been going to disappear such as the withdrawing of a commercial center. Most of these residential areas had been developed under the theory of neighborhood unit [1], but their structures have changed into a surrounding that cannot provide commercial services within realistic walking distance due to various factors such as population outflow and aging. Especially, traffic convenience of some areas has seriously declined. In these housing estates developed at suburban slope area, riding a bicycle is not realistic because even a short distance movement burns a lot of power. Similarly, footing from a resident's house to a destination such as a bus stop has also probably been difficult. Unlike flat residential area, perhaps the traffic convenience in slope housing estates is considerably poor. Also, the traffic convenience strongly depends on the locational conditions such as the distance to bus stop and with/without private cars. Now, with the downward turn of a population, the local government having such these surroundings should address to improve suburban traffic convenience. Most of all, along with the additional decrease of Japan's population and the society aging, the living environment of the suburban slope residential estates is getting only worse for elderly people. If such a situation goes on, elderly people will be isolated and left out in these areas as "vulnerable road users". We should examine a new public transportation system which effectively utilizes idle transportation resources such as private car and keeps the residential service level for the longest possible time.

Our previous study [2][3] showed the concept of information sharing system to support the daily life of residents living in a place where public transportation is not sufficiently developed. The previous study [2] gave the investigation result of residents' traffic awareness in the suburban slope residential areas at Itsukaichi district, the main bedroom suburb of Hiroshima City. The previous studies [3][4] embodied the concept of a transportation support model for vulnerable road users in Itsukaichi district and proposed a Web service. In this paper, based on the investigation results on residents' traffic attitude in the slope residential estates at Itsukaichi District, Hiroshima City, and also the examination of previous studies, we develop a web service to support vulnerable road user's daily life in suburban residential estates, mainly elderly people. The service of the proposed system is designed by the concept of sharing economy [5][6]. We have considered that an efficient method to embody the idea of transportation support model would be the sharing of each resident's schedule to move with Web technology. Only municipal public transportation services cannot solve the vulnerable road user's issues enough. Thus, the proposed system aims to provide a transportation means which is a service giving last one-mile movement with local resident's help. The core service of the proposed system is to enhance the encounter between local community people which is created by personal information sharing such as each one's schedule, occupation, address, skills, and troubles in daily life, and will lead to solving a troubled resident's problem related to the movement for daily life. In this paper, this encounter is called as "mobility-sharing". The proposed system provides primary two services, the time-dependent skill/trouble information sharing and the negotiation support with a thread based BBS, both of which enhance the mobility-sharing and make the cooperation between local community residents easy. Concretely, the former is to facilitate someone's request and acceptance, and the latter is to realize the mobility-sharing such as ride-sharing from a residential area to a city center. With the proposed system, for example, a resident, who will move to his/her destination with one's private car, can help a trouble of another resident (mainly elderly people) with his/her original purpose together. Most of the troubles will be the ride-sharing with private transportation means and the conveyance of luggage 


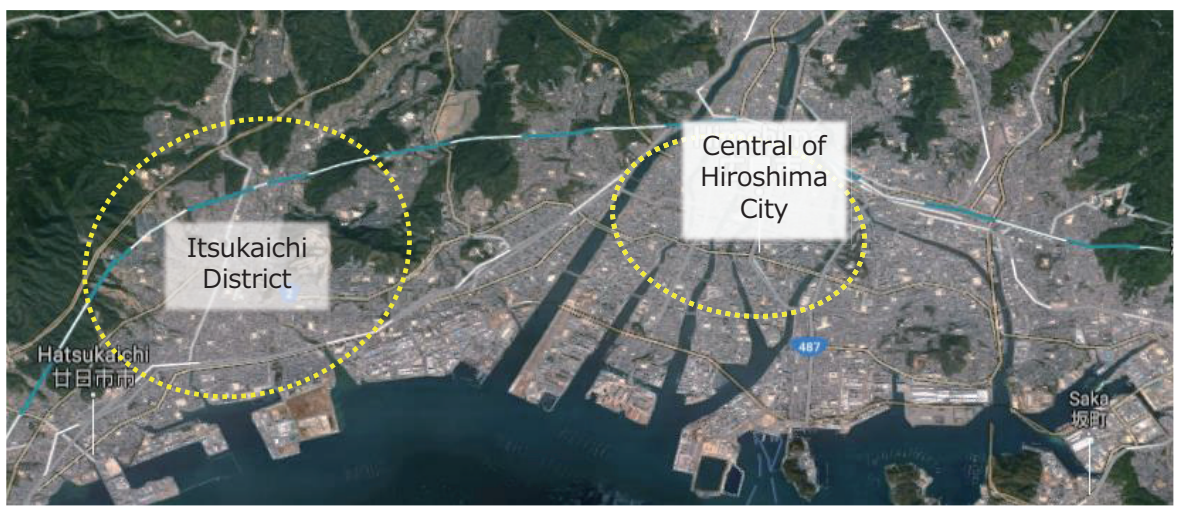

Figure 1: Itsukaichi district

from residential areas to a place near another resident's destination or vice versa. However, the targets are not limited to these and include troubles in daily life such as gardening, dog-walking, and maintenance of electric appliances. We expect that the mobility-sharing generated by the proposed system leads to enhance local people's relationship building and the regeneration of local community.

\section{Current situation of transportation means in Itsukaichi Dis- trict}

Itsukaichi District, the target region of this paper, is a major bedroom suburb of Hiroshima City as shown in Figure 1. In Itsukaichi District, there are some housing estates systematically developed. However, these housing estates are far from the central area and inconvenient for people who cannot freely use his/her private car called vulnerable road users. Additionally, footing and riding bicycle are not easy because most of these housing estates are at the slope area on the surrounding mountains of the city. Therefore, some of vulnerable road users already emigrated from these housing estates to apartment buildings in central areas convenient for shopping and transportation. The current condition of Itsukaichi District is shown in Figure 2.

The authors investigated the actual situation of transportation in Itsukaichi District from the questionnaire for 2951 households at some regional districts. The result clarified what the local people had been dissatisfied with a routine short-distance move like shopping [2]. The targetted districts have no trains connecting each residential area with each major shopping store, and also few buses. Moreover, the distances to bus stops are not close because most of the bus stops are located at main roads. This questionnaire was composed of some items to investigate the actual situation in everyday move and needs for transportation means. The investigation items were such as questionee's awareness about the current public transportation (have been satisfied/unsatisfied, and its reason).

The first aim of our survey was to unveil the real consciousness according to age-groups for current transportation service at each residential area. The survey showed the ratios of daily transportation means of seniors (over 60) and non-seniors in each residential area. It suggested that the use of one's private car is not easy for seniors compared with non-seniors. The main transportation means of people in these residential areas will further change from private car to public transportation along with aging. Therefore, it is important to examine 


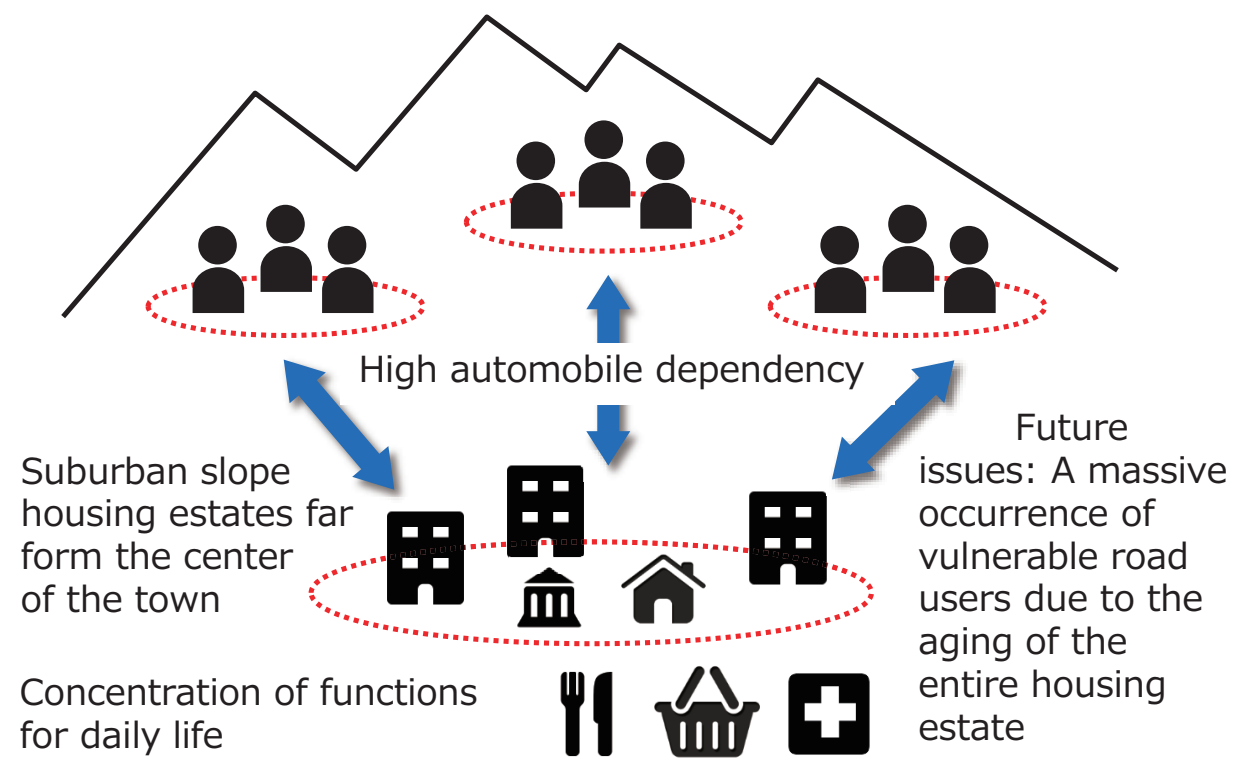

Figure 2: The current condition of Itsukaichi District

the ideal state of current transportation service and its improvement including the consideration of future conditions. From the survey result on seniors'/non-seniors' satisfaction level of transportation in each residential area, we saw the fact that seniors had not been satisfied with the current transportation service than non-seniors even the volume of gaps was different depending on the residential area.As the requirement for reducing the frustration on transportation, seniors in some residential areas tended to desire the improvement of bus service. The survey result also showed that these seniors have also requested a convenient and accessible transportation service different from present usual bus service.

Our previous survey confirmed the difficulty in the everyday movement for these residential areas. The survey also unveiled the situation that many of the residents who intend to go out had strongly depended on their private car due to the reason that there had been few public transportation near their living areas. Some areas have had no bus routes within reasonable walking distance, and most of the residents in these areas had disgruntled about the low service level of public transportation more than the other regions. However, introducing a public transport service must be unrealistic because there are only a few residents in such areas and such a surrounding is unprofitable. Thus, the authors have started to examine a realistic plan for these depopulated areas to improve the service level of transportation by introducing a new kind of transportation service. As a new idea of public transportation, usually, a demand responsive bus or a community bus is mentioned. All residential areas showed a lot of positive replies for introducing community-based new transportation means like these bus services, and especially, seniors tended to be higher than non-seniors. Some local governments operate community bus which travels throughout residential area and also stops by main places of transportation such as bus stops, shopping stores, and rail stations, but most of these are money-losing. Therefore, the authors consider that we have no choice to improve the service level of transportation except the mutual aid between residents. 

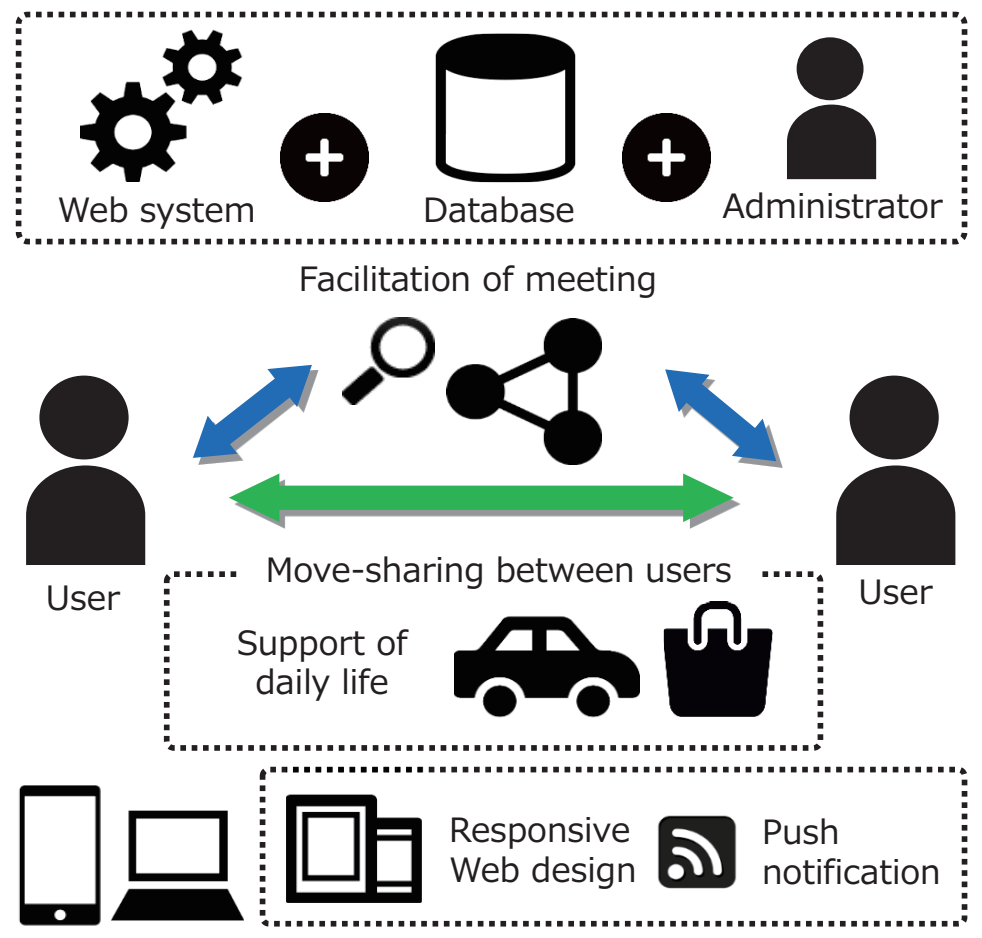

Figure 3: The concept of the proposed system

\section{Proposed System}

\subsection{Concept}

The concept of the proposed system is shown in Figure 3. The structure of the proposed system in this paper follows the device configuration [7] already published as a patent. The proposed system creates an opportunity of mobility-sharing between two or more local people. With this encounter, for example, a troubled person, who has no daily transportation means but wants to go out, can find a person, who lives near this troubled person and will be able to bring the troubled person along together with his/her out. That is, the main aim of the proposed system is to facilitate community-based mutual aid in the movement of everyday life and to deal with the various and small kinds of demands in daily move finely by the meeting of people who go out in the same direction. Although the mutual aid is volunteer activity, a helper can obtain decent wages as a donation, and the cost will be gathered from the user's system usage fee.

The main targets of the proposed system are two kinds of mobility-sharing: the one is ride-sharing where two or more residents go somewhere together by a resident's car after adjusting each one's schedule, and the other is conveyance where a resident helps another resident's conveyance by his/her moving. The flow of mobility-sharing is, firstly, a troubled resident called as "client" posts a request to the proposed system. For example, a resident, who wants to go out but does not have transportation means or who needs assistance for a conveyance of daily necessaries, inputs the detail to the proposed system. Secondly, if another user, called as "server", who has a willingness to accept another resident's help, checks the details of another resident's request such as conditions and personal information and judges it acceptable, the server can contact the client by using direct message function. 


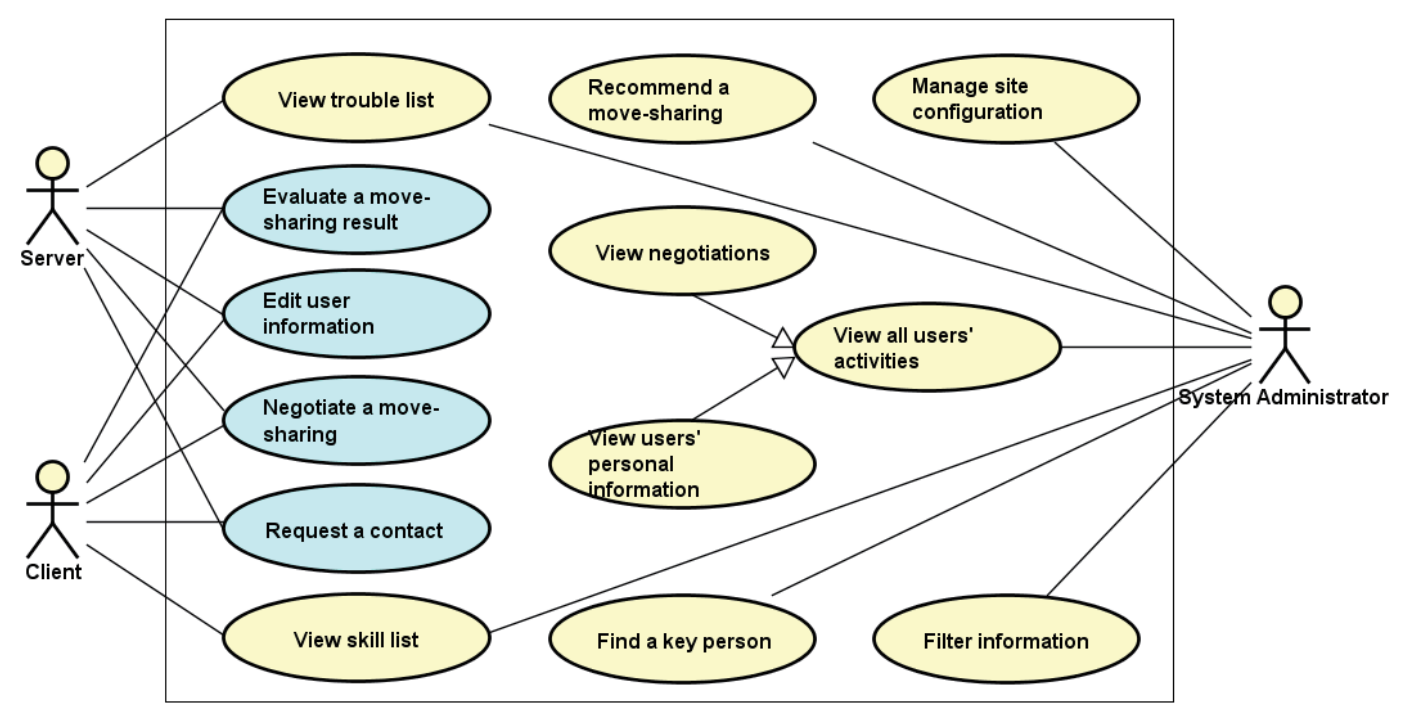

Figure 4: Use cases of the proposed system

After the negotiation with the direct message function, these residents decide mobilitysharing if they reach an agreement. The proposed system will promote the rationalization and the efficiency of people and good's movement. Furthermore, Since the proposed system encourages the meetings of each local resident, the authors expect that the proposed system can contribute to reproducing a local community.

There are already some ride-sharing services in Japan. For example, a ride-sharing service named "notteco" has already been started in Japan, and the main target of this service is long-distance travelers. Similarly, a ride-sharing named "nori-na" 2 has also operated. After a user registers the detail of travel, such as route, capacity, and price, all user can check the information. Typical ride-sharing service assumes cooperation realized by a negotiation between users. In addition to usual identification, as safety measure, users require personal information such as the authentications using the mobile phone number, evaluation from others, or external SNS. This service does not fall under any of the Article 80 of the Road Transportation Act because those who receive the service do not pay a fare but bear only a part of the total cost for trip. The proposed system can be realistic as a business by adopting the same operational policy with these existing ride-sharing services. In these ride-sharing services, a driver can get a fee for assistance in a different way from fare because getting fare is illegal except the approval by the Minister of Land, Infrastructure, Transport and Tourism. Then, the proposed system will give an incentive like "local point" to the effort of the server. Each user can exchange the local point to a gift ticket or can receive a service in the local area. The proposed system is operated by the system usage fee from all users, where the price of the usage fee is decided depending on the usage frequency (mainly the number of requests for the servers).

\footnotetext{
${ }^{1}$ notteco, http://notteco.jp/

${ }^{2}$ nori-na, http://www.norina.jp/
} 


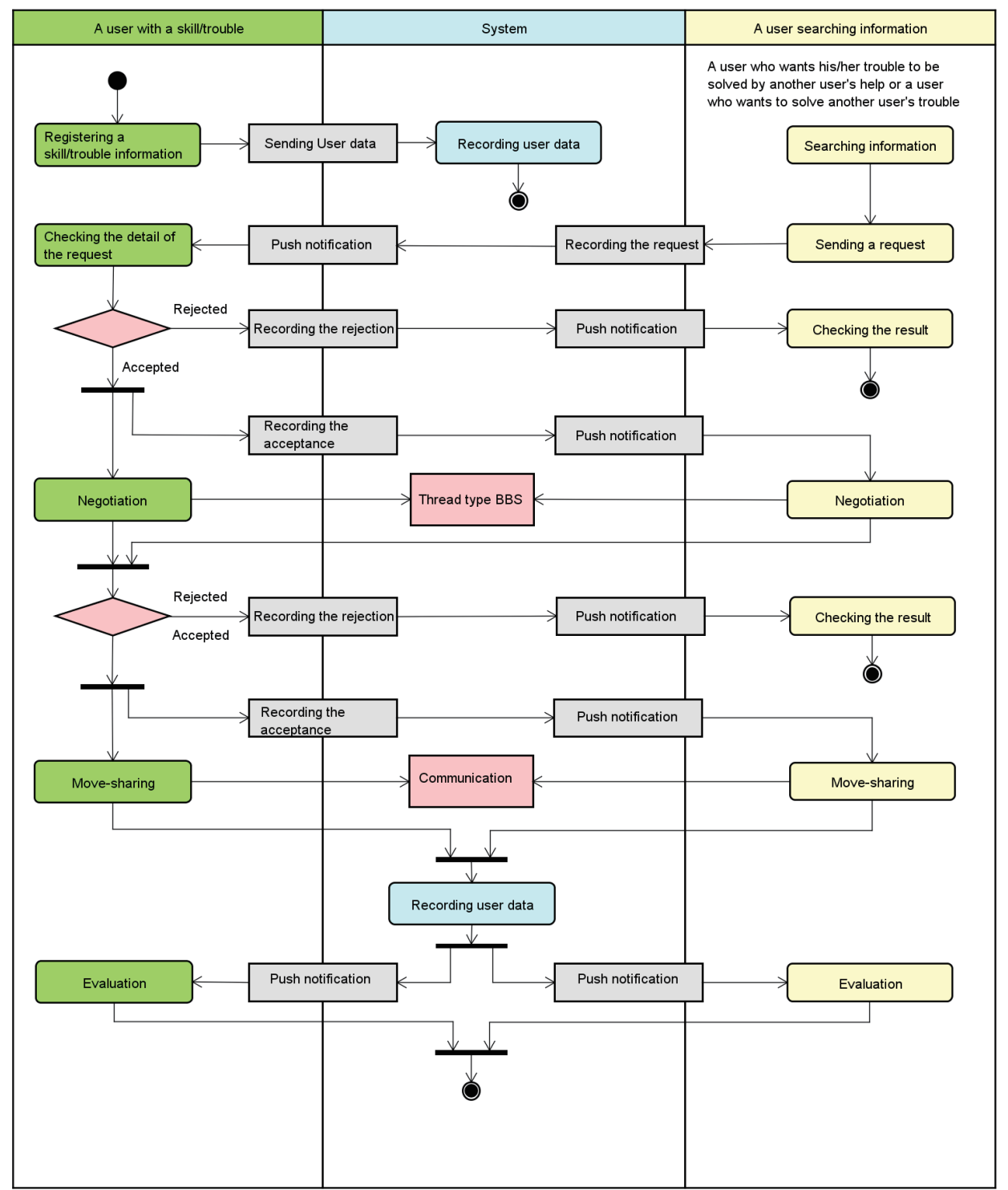

Figure 5: The flow of activities

\subsection{Functions}

The use cases of the proposed system are summarized as shown in Figure 4. To support the cooperation between local community residents easily, the proposed system provides two primary functions: negotiation expressed by the use case "Negotiate mobility-sharing", and skill/trouble sharing expressed by the use cases "View trouble list" and "View skill list". For example, as the types of the demands, the one may be mainly ride-sharing with the other resident's mobility, and the other may be the conveyance of luggage from residential areas to a place near another resident's destination or vice versa. The proposed system has three types of users "server", "client" and "system administrator". The client is a user with trouble in daily life, and vulnerable road users are assumed as the main target of the client. "Server" is a person who can solve a client's trouble. "System administrator" is the administrative staff of the proposed system and is a user who can grasp the activities 


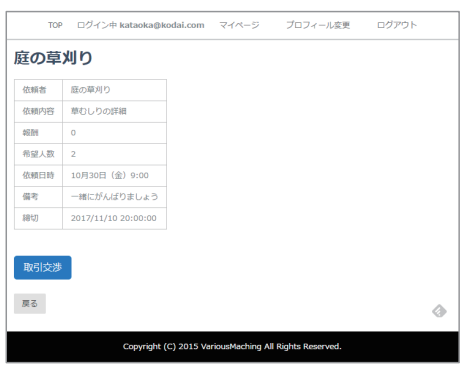

Detail of trouble

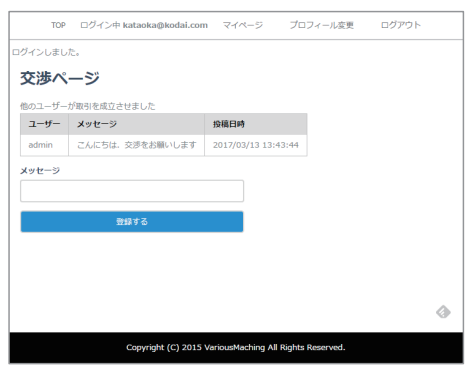

Thread type BBS

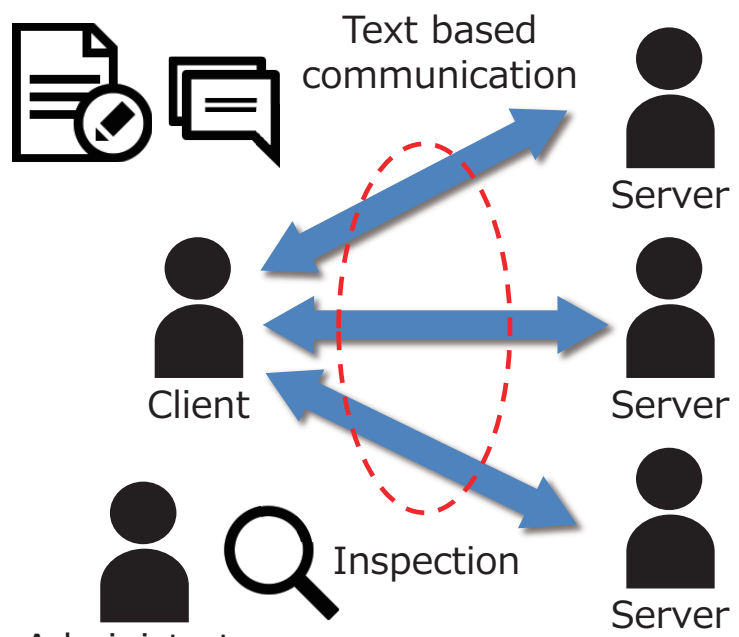

Administrator

Figure 6: The flow of activities

of all users. The mediation of the system administrator is possible to prevent troubles between users and the illegal use in advance. To realize the mediation, the proposed system provides the use case "Filter information" to eliminate illegal and inappropriate information and the use case "View all users' activities" to see the activity among users for the system administrator. A user can use the system after registering user information and creating an account with the use case "Edit user information" Each server registers his/her skill (mainly the schedule of travel) in advance. Similarly, each client registers a trouble. In this system, it is available to register more than one skill and trouble at the same time. The client can negotiate ride-sharing with multiple servers at once as shown in Figure 6.

Since the information registered by a user is added to the skill/trouble list, all users can view the information on all users' skills/troubles. The skill/trouble list is also available for those who do not have an account, but creating a user account is necessary to view detailed personal information or to contact other users. When a user searches the skill/trouble list and finds a skill/trouble satisfied with the user's condition, the user can contact the owner of the skill/trouble, where the flow is shown as the use case "Request a contact". After this action, if the contact is accepted, these users can negotiate by text message on the secret page, where the flow is the use case "negotiate mobility-sharing". The system administrator monitors all actions of the negotiation and mediates when necessary. After the negotiation, if the condition of mobility-sharing is satisfied, the server helps the client in a real field by keeping in touch with each other such as by phone (the phone number is published after the 


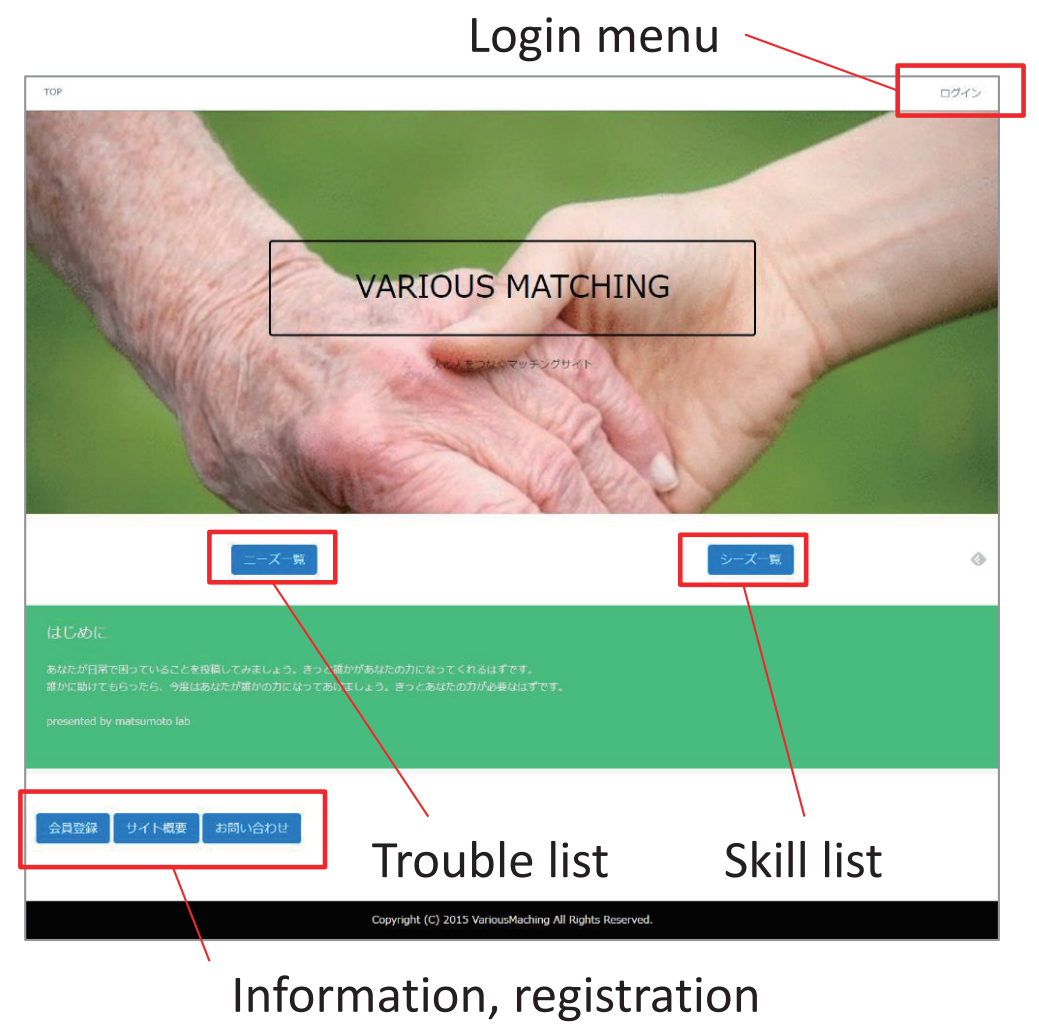

Figure 7: Top page of the proposed system

formal decision of mobility-sharing). After the completion of mobility-sharing, both the server and the client give the mutual evaluation. The flow of the above activities is shown in Figure 5. This activities flow is a summary of three use cases. As shown in Figure 5, push notifications are made by e-mail every time at the important timing.

In this system, the system administrator has multiple roles. Firstly, in the use case "View all users' activities", the system administrator appropriately checks and sees the condition of all negotiations and the details of all users such as what and which kind of skills and troubles each user has. Also, the system administrator can also analyze the user's evaluation after mobility-sharing and also the relations between users. Based on the analysis data, proposing a mobility-sharing and finding a key person will be realized in the use cases as shown "Recommend a mobility-sharing" and "Find a key person" respectively.

\subsection{Implementation}

The proposed system was constructed by PHP 5.4.9-4, and its requirements are Linux kernel 3.8.0-27, Apache 2.2.22, MySQL 5.1.54, and Fuel PHP 1.7.2. Since the user interface was implemented by jQuery 2.0.2 and jQuery Mobile1.3.2, the proposed system can provide rich user experience with responsive Web design. Figure 7 shows the top page of the proposed system. The site construction of the proposed system is shown in Figure 8. The constructions of skill/trouble lists are same, so Figure 8 only shows the detail of the skill list. Each user can access the management page after login operation. When creating own page, the user has to use the management page. The skill/trouble management function can show all items as list format registered by the user. Each user can negotiate with two or more 


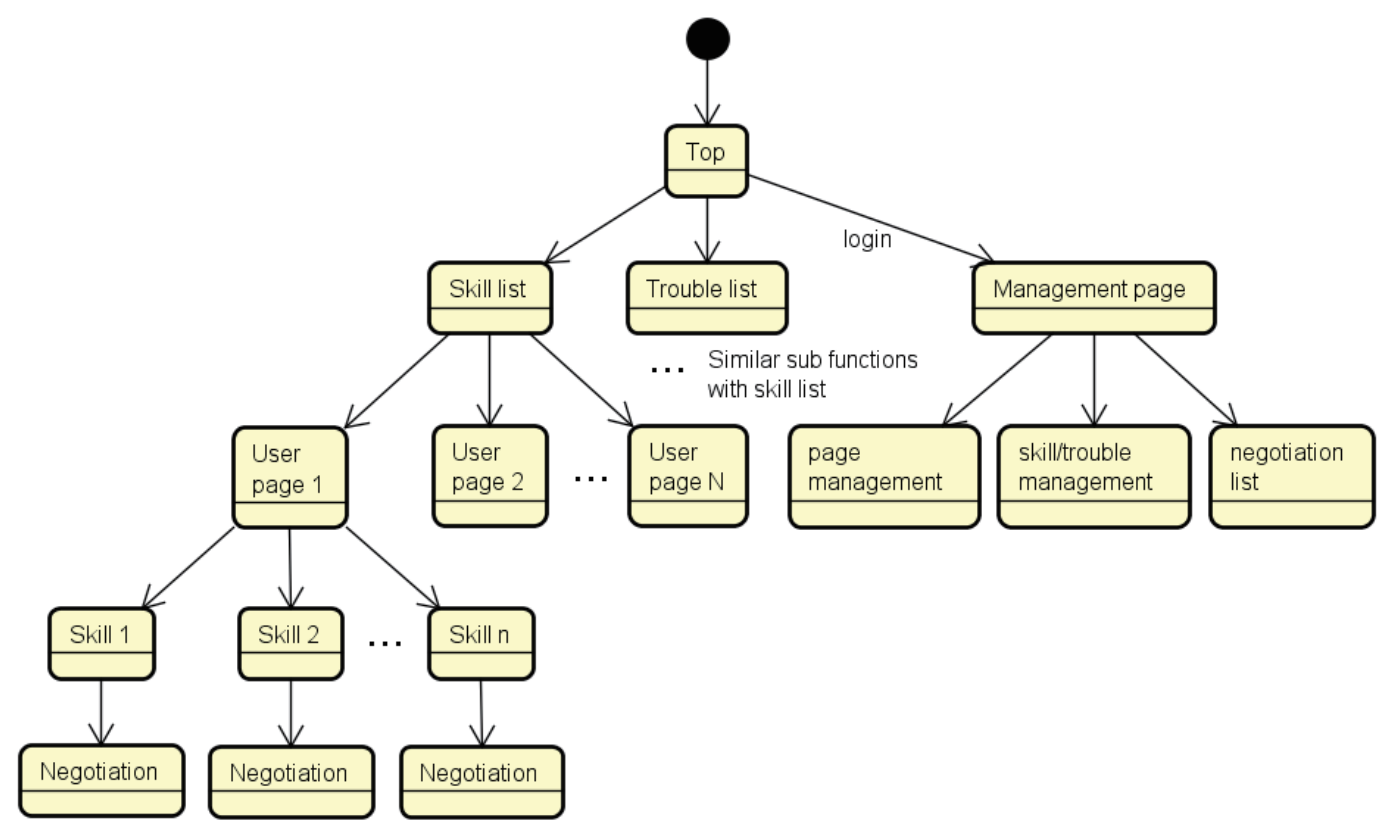

Figure 8: Site constitution

other users at the same time, and each will be managed in the negotiation list. Also, each user can register one or more skills or troubles. Therefore, each user page contains some skills/troubles. The function for requesting negotiation is prepared on the page showing details of these skills/troubles. The operation examples of the proposed system are shown in Figure 9.

\subsection{Organizational Frameworks for Solving Regional Transportation Prob- lem}

In order to resolve such the social issues in local community, mainly the issue on vulnerable road users, and maintain/vitalize the local community, FIVE (Fellowship for Itsukaichi's Vitalization and Enrichment) ${ }^{3}$, a non-profit organization, was organized by volunteers for contributing to promote the public benefit of the Itsukaichi District. The present primary task of FIVE is to propose and implement the policy to activate more the commerce, the life, the education, and the traffic, which can respond to an aged society. The scheme of FIVE is shown in Figure 10. FIVE have been creating the opportunity to connect a senior to the local community and also making the place to offer information assumed to be required for seniors. The proposed system will be operated under the management of FIVE in the near future, and the authors will continue reporting the obtained result continuously.

\subsection{Previous Studies}

There are few studies on personal/mini transportation means for supporting resident's movement. The contributions of most of these studies have focused only on the discussion about the concept or the design methodology of transportation services based on individual travel

${ }^{3}$ FIVE, http://www.city.hiroshima.lg.jp/www/contents/1384995381935/ 


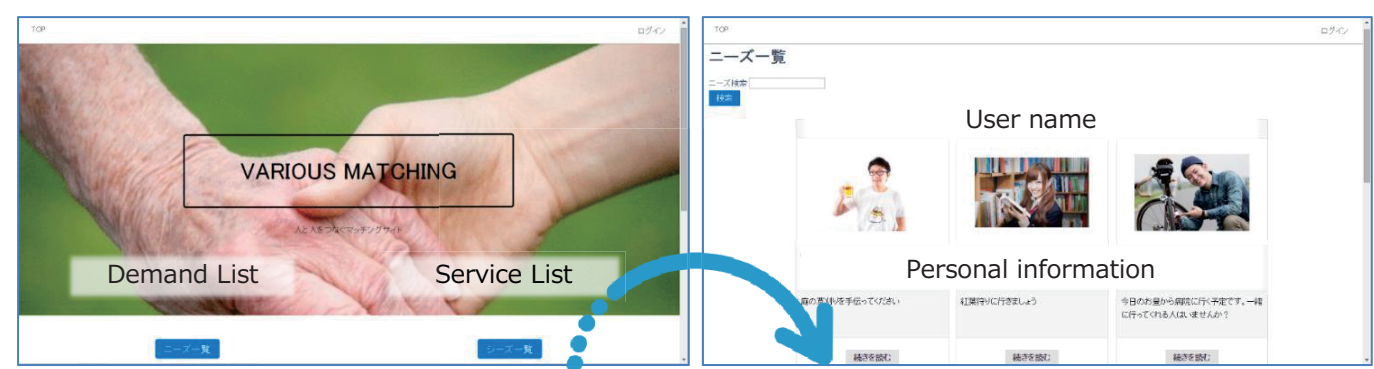

(a) Top page

(b) User list (in order of arrival)

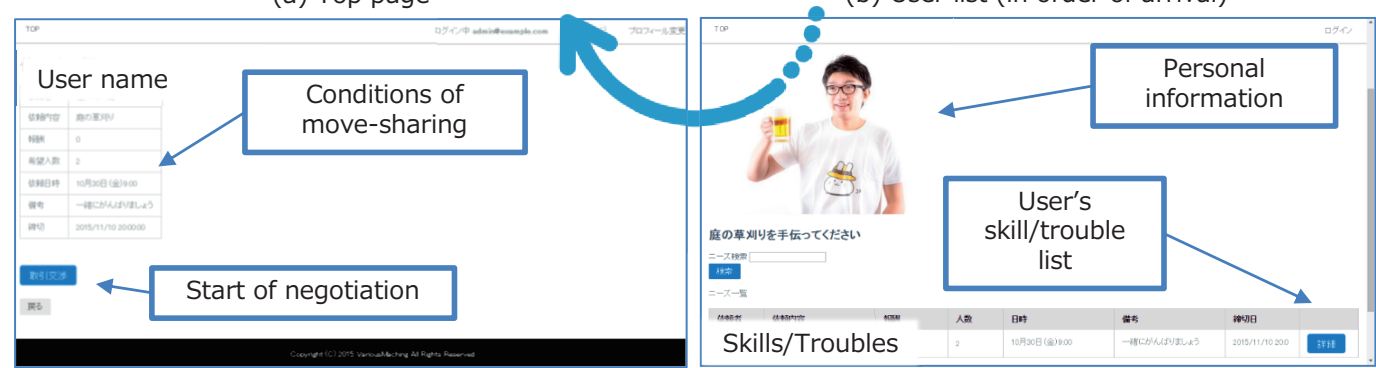

(d) Negotiation page of move-sharing

(c) Personal information

Figure 9: Operation examples

means [8]. On the other hand, some studies have reported the simulation models introducing a transportation service for local city and its neighboring parts [9][10]. For example, Kleiner et. al. presented a model of multi-agent dynamic ride-sharing model [11]. Their systems allowed to trade off the minimization of vehicle kilometers traveled with the overall probability of successful ride-shares. Agatz et. al. showed the optimization challenges that arise when developing technology to support ride-sharing and surveyed the related operations research models in the academic literature [12].

The effort of this paper differs from these usual studies in the point that this paper proposes a structure available for supporting vulnerable road users on the basis of the conscious survey result of the actual movement [13], and aims to solve the transportation problem with self-help, mutual assistance, and public assistance within the local region. The contribution of this paper is placed as the effort to embody the concept such as "Town development company"[14], which has recently been actively advocated.

\section{Evaluation Result}

Seniors who are not good at IT can indirectly use the system with the help of a visiting nurse, a social worker, or a staff member of FIVE. Therefore, in order to confirm the usefulness of the proposed system, it is necessary to clarify the evaluation of mobility-sharing itself more than to clarify the operability of such as UI and UX. Since the existence of seniors' demand is obvious from the investigation of the previous study [3], we should examine the expectation for the user's proposed system to use as the server. Especially, participa-tion by young people must be indispensable for activating mobility-sharing. Therefore, we conducted a questionnaire to Hiroshima residents from 20 years old to 24 years old and obtained the evaluation of the proposed system. Each response of the questionnaire was on Likert scale 4 points (strongly agree, weakly agree, weakly disagree, strongly disagree); 4 is the maximum (positive), and 1 is the minimum (negative). Each experiment participant 


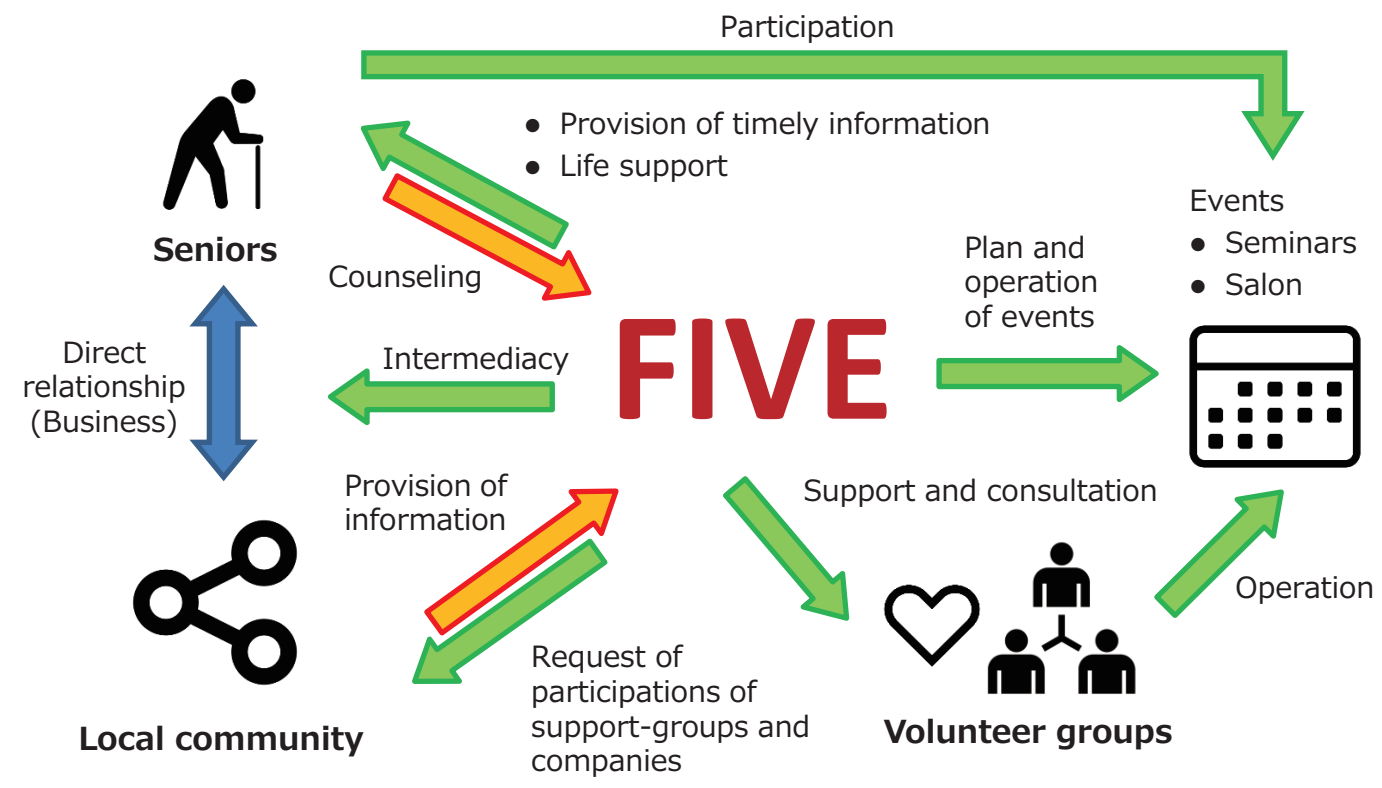

Figure 10: Scheme of FIVE

manipulated the prototype of the proposed system for a sufficient time while receiving an explanation about each function from an experimenter in charge after listening to about 10 minutes presentation on the proposed system. After that, each experiment participant answered each question of the questionnaire. First of all, in order to confirm the reliability of the questionnaire result, we confirmed whether the subjects sufficiently understood the concept and idea of the proposed system by the first question. As a result, 42 respondents who gave 3 or more evaluation to this question were used for analysis. The items of the questionnaire on the evaluation of the proposed system are as follows.

- Q.1: Did you understand the concept and idea of the proposed system?

- Q.2: When the proposed system is actually launched, do you want to use it as a server?

- Q.3: When the proposed system is actually launched, do you want to use it as a client?

- Q.4: What do you think as a general opinion on the mutual assistance of local people by ride-sharing?

- Q.5: Do you predict that ride-sharing will be accepted and used by local people?

- Q.6: What do you think as a general opinion on the mutual assistance of local people through the support of shopping and transport of goods?

- Q.7: Do you predict that supporting shopping and transport of goods will be accepted and used by local people?

- Q.8: Do you consider that the function of the proposed system is sufficient? 


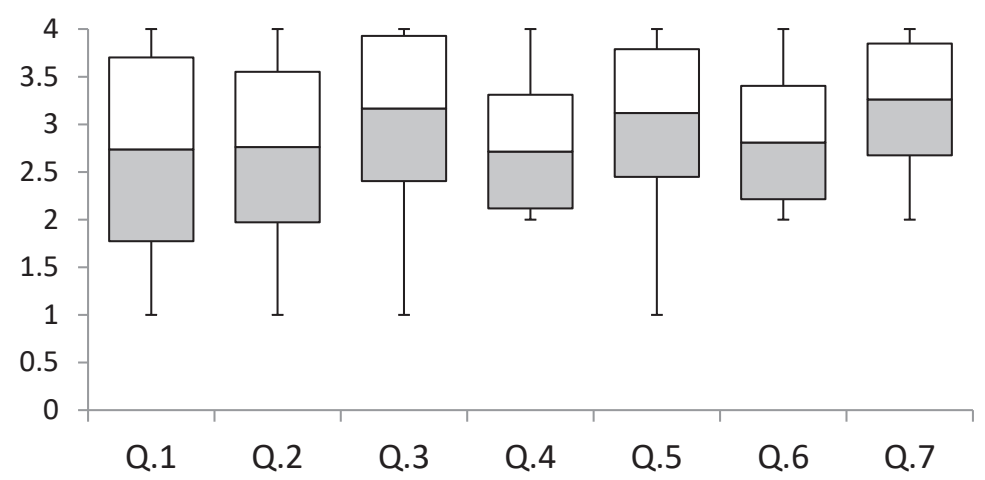

Figure 11: Summary of the evaluation result on the basis of Likert scale 4 points, Min, Ave-SD, Ave, Ave+SD, Max

Q.2 and Q.3 are evaluations as to whether experiment participants actually wanted to use the proposed system. Q.4 and Q.6 are evaluations as to whether experiment participants thought the proposed system would be good as a general business from an objective standpoint. Q.5 and Q.7 were based on the subjective evaluation of experiment participants. Q.8 is one's impression after manipulating the prototype of the proposed system. Summary of the evaluation result is shown in Figure 11. Boxes represent standard deviation, upper and lower lines represent maximum and minimum, and centers represent average. From Figure 11, we can confirm the high evaluation overall. Especially the evaluations of Q.4 and Q.6 were high. Based on the result, young people would be highly interested and confirmed high expectations for the proposed system. However, contrary to the high evaluation of Q.4 and Q.6, the evaluations of Q.5 and Q.7 were relatively low. This result would be evidence that the experiment participants think the proposed system socially good but they felt that the proposed system includes various problems.

From experiment participants who answered low evaluation (weakly disagree, strongly disagree) about the function of the proposed system in Q.8, the following opinions were presented as functions that the proposed system should provide.

- Authentication of the server (recommendation from another resident to the server).

- Introduction of reliability factor to eliminate irresponsible service provision. For example, suspending people with low reliability.

- Communication between users.

- Limiting an unspecified number of users, but only for use by users who are connected by SNS.

- Personal information inquiry.

Regarding the reason for the evaluation of Q.2, we got answers from multiple selection methods. Positive reasons (abbreviated as P. R. 1-4) are as follows, and these summaries are shown at the left side in Figure 12.

\section{Positive reasons}

- P. R. 1: I tend to be interested in a new service. 

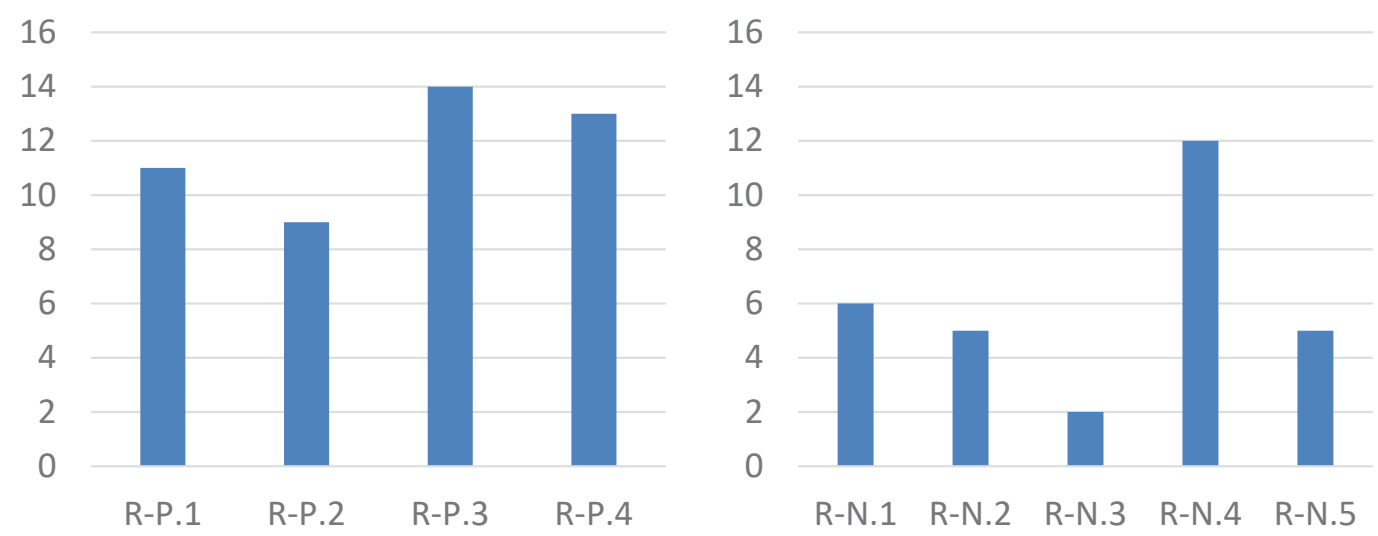

Figure 12: Reason for the evaluation of Q.2

- P. R. 2: I want to contribute to the local community.

- P. R. 3: I want to make a new connection with people in the local community.

- P. R. 4: I want to get rewards.

Similarly, negative reasons (abbreviated as N. R. 1-5) are as follows, and these summaries are shown at the right side in Figure 12.

\section{Negative reasons}

- N. R. 1: I do not go out much.

- N. R. 2: I do not want to disclose personal information.

- N. R. 3: I do not want to drive a car/I do not use a car.

- N. R. 4: I feel troubled to get involved with others.

- N. R. 5: I feel annoying to engage with others.

"N. R. 4" was remarkably larger as the reason of negative opinion than others. This result suggests that most of the people living in the same area are "unknown people" for the experiment participants, and it is great psychological resistance to be involved with such other people. It is thought as a cause that the low level of daily relationship with the local community is behind the opinion. On the other hand, there were positive opinions such as "I want to engage in the local community", or "I want to earn rewards". While the opinion of earning reward is convincing because it is a general answer, the result of "P. R. 3" is surprised fact because there would be many young people who want to engage more with the local people and also are more willing to contribute to the activation of their local area despite the decline of local community. This opinion is opposite of the opinion "N. R. 4", so it seems young people's consciousness about local community is polarized between high and low.

Regarding the reason for the evaluation of Q.3, similarly, we got answers from multiple selection methods. Positive reasons (abbreviated as P. R. 5-7) and negative reasons (abbreviated as N. R. 6-9) and this summary are following and in Figure 13. "P. R. 7" would 

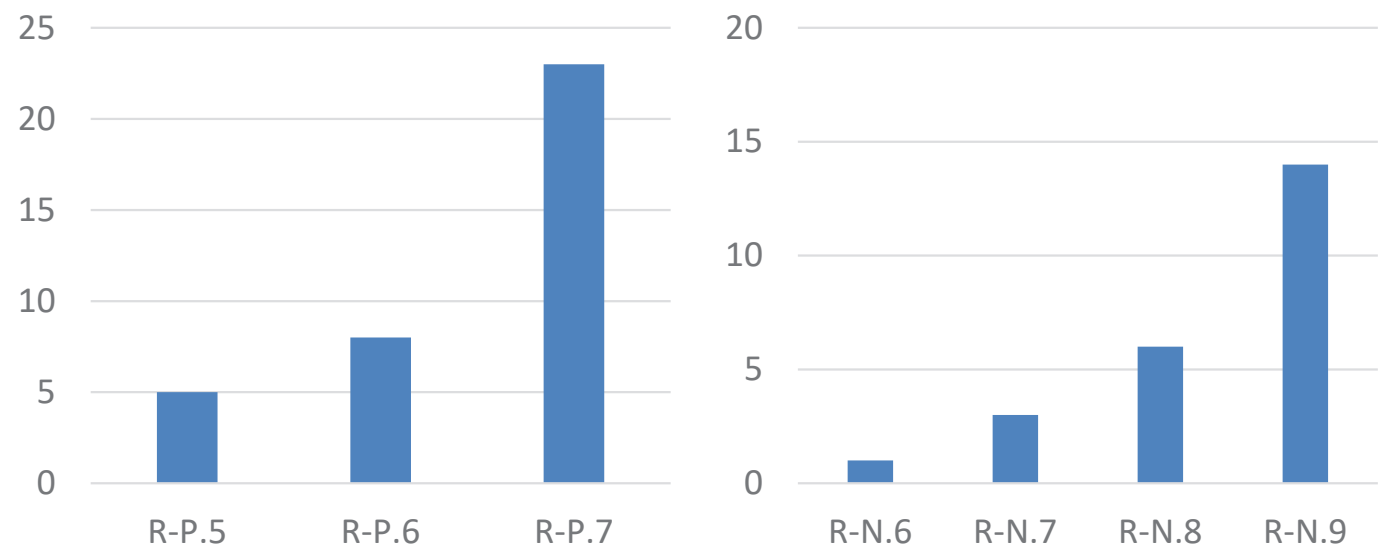

Figure 13: Reason for the evaluation of Q.3

imply that even young people are feeling a little inconvenience to move. As the negative opinion, the value of "N. R. 9" is remarkably high. Although traffic accidents can generally happen to anyone, some experiment participants were sensitive to traffic accidents. In addition, they are very sensitive to troubles among users even though troubles in daily life are extremely rare. As this reason, we consider that they do not trust other people because they have little experience cooperating with local people. This would be a remarkable proof that some of the young people are independent of their area.

\section{Positive reasons}

- P. R. 5: I want to reduce waste.

- P. R. 6: I want to interact with local people.

- P. R. 7: I feel that the proposed system is useful.

\section{Negative reasons}

- N. R. 6: I am worried about riding unknown person's car.

- N. R. 7: I am not interested in the local area.

- N. R. 8: I do not need help of people because I can do my own thing myself.

- N. R. 9: I am worried about traffic accidents and troubles among users.

In addition to the responses of above-mentioned items, we also obtained the consciousness and the actual condition of daily trips for each experiment participant by the following two items.

- How often have you participated in local events?

- How often have you got involved with locals, especially the elderly and children? 
Above these frequencies were evaluated on Likert scale 4 points (strongly agree, weakly agree, weakly disagree, strongly disagree). We interpreted these answers as the degree of closeness with their local area. We calculated the sum of the evaluation values of these two items, and divided experiment participants into two groups depending on the sum; the one is a group with the value of 3 or less (assumed as the group distant to local area, $\mathrm{N}=26$ ), and the other is a group with the value of 4 or more (assumed as the group close to local area, $\mathrm{N}$ =16). After that, the average of the evaluation values was calculated according to groups, and the difference between them was analyzed. The results are shown in Table 1. There were differences in some items, but no significant differences. The group close to local area tended to highly evaluate overall. On the other hand, the ride-sharing service was skeptical as the people closer to their local area, and also, the functions of the proposed system were felt to be inadequate. People closer to their local area know more about the current local state. Therefore, we understand this result as that they judged the proposed system should include more functions to fully realize their daily relationships with their local community. About the point that was skeptical about the ride-sharing service, we consider that they knew home delivery is actually desired for the local area rather than ride-sharing.

Same with the degree of closeness to his/her local area, we also calculated the closeness about the frequency of opportunities to help others by sharing resources in everyday life. We additionally obtained the responses from experiment participants by the following two items.

- How often have you shared travel means such as taxis with multiple people?

- How often have you done mutual aid for daily shopping (you have supported others' shopping / you have had help of shopping from others)?

We summated above-mentioned two evaluation values, and divided experiment participants into two groups; the one is a group with the value of 3 or less (assumed as the group with low attitude to mutual aid, $\mathrm{N}=23$ ), and the other is a group with the value of 4 or more (assumed as the group with high attitude to mutual aid, $\mathrm{N}=19$ ). The results are shown in Table 2. Table 2 shows an interesting trend. First, since there were significant differences $p$ $<0.01$ in Q.4 and Q.6 by Welch's t-test, people with more frequent interactions strongly sympathized with the proposed system, which is a reasonable trend. On the other hand, the evaluations of the group High at Q.5 and Q.7 were lower than the group Low. This would mean that the evaluation of the proposed system itself is low for people who regularly help each other. As one of the reasons for this result, many people of group High would think the proposed system is unnecessary because the local community has already been established and they are always possible to share resources in everyday life. If so, it is not a negative result because the proposed system targets areas where the local community is declining. On the contrary, if we thought that regional cooperation could not be realized simply by some sort of system such as the proposed system, this is a tough result. However, regarding the popularization of the proposed system, we plan to operate it with FIVE. Therefore, we are not trying to realize our purpose, regional cooperation, by only the proposed system. We position the proposed system as a system that supports people working for their local area. We remind that it would be essential to closely cooperate with activity groups for local communities for promoting the proposed system. 
Table 1: Difference in evaluation (Ave.) according to distance with local community

\begin{tabular}{c|cccccccc}
\hline Group & Q.1 & Q.2 & Q.3 & Q.4 & Q.5 & Q.6 & Q.7 & Q.8 \\
\hline Distant $(N=26)$ & 3.69 & 2.65 & 2.69 & 3.12 & 2.73 & 3.08 & 2.77 & 3.31 \\
Close $(N=16)$ & 3.87 & 2.87 & 2.87 & 3.20 & 2.67 & 3.20 & 2.87 & 3.13 \\
\hline
\end{tabular}

Table 2: Difference in evaluation (Ave.) according to frequency of mutual aid in daily life $(* *: p<0.01)$

\begin{tabular}{c|cccccccc}
\hline Group & Q.1 & Q.2 & Q.3 & Q.4 ** & Q.5 & Q.6** & Q.7 & Q.8 \\
\hline Low $(N=23)$ & 3.70 & 2.78 & 2.74 & 2.87 & 2.74 & 2.83 & 2.83 & 3.26 \\
High $(N=19)$ & 3.84 & 2.68 & 2.79 & 3.53 & 2.68 & 3.47 & 2.79 & 3.26 \\
\hline
\end{tabular}

\section{Conclusion}

This paper gave the detailed description of the scheme of transportation support model shown by our previous work as the proposed system and developed its prototype. This paper focused on creating an opportunity for ride-sharing of a short trip such as a move to a city center, and for sharing of luggage transportation while a user moves, and these sharings defined as mobility-sharing. This paper assumed the core service of the proposed system as facilitation of someone's request and acceptance of mobility-sharing, and clarified details of the proposed system's use cases, activities flow, user interface. Since the proposed system can support the cooperation between local community residents easily, so we expect the proposed system will enhance people's relationship building and the regeneration of local community. In the future, the pair of mobility-sharing will be automatically decided, and the result is provided to each user's smartphone with push notification.

Based on the evaluation result, local young people were highly interested in the proposed system and evaluated the proposed system socially good, but they felt that the proposed system includes various problems. From the reason for evaluation of the question "When the proposed system is actually launched, do you want to use it as a server?", the experimental result showed surprising fact that there would be many young people who have wanted to engage more with the local people and also been more willing to contribute to the activation of their local area despite the decline of local community. On the other hand, there were many opinions like "I feel troubled to get involved with others". From this result, we concluded that young people's consciousness about local community is polarized between high and low. From the reason for the evaluation of the question "When the proposed system is actually launched, do you want to use it as a client?", we considered that local young people do not trust other people because they have little experience cooperating with local people. This would be a remarkable proof that some of the young people are independent of their area. The group of local young people close to the local area had positive impression for the proposed system but evaluated the ride-sharing service low. They judged the proposed system should include more functions to fully realize their daily relationships with their local community. Local young people who regularly help each other evaluated the proposed system itself relatively low. We considered that they would think the proposed 
system is unnecessary because the local community has already been established and they are always possible to share resources in everyday life. However, since the proposed system targets areas where the local community is declining, we thought this reaction was not so serious. Still, we reaffirmed that it would be essential to closely cooperate with activity groups for local communities for promoting the proposed system.

We may see some radical changes in transportation in the near future. For example, a new technology represented by "automatic driving" may be put to practical use soon. Such a new technology may drastically change the forms of owning and using private cars. Also it may change the division of roles between private cars and other transportation means such as taxis and rental cars. Dependence on private cars is relatively heavy in Hiroshima region. It has limited flat land for its population. Especially suburban areas are mostly hilly, and there are many areas which are quite unsuitable for walking and riding bicycles. In the urban area of Hiroshima City, which has a population of 1.5 million, it is financially difficult to have highly-developed public transportation systems such as the railroad. In the current aging society, there will be more and more people who cannot make use of private cars, and in Hiroshima region which heavily depends on private cars, there will be more and more areas in which elderly people find it difficult to live a comfortable life in terms of daily traffic. In this light, it is important to consider viable plans to cope with such problems. The main aim of this paper is to propose systems to support the transportation of people and things, as mentioned in Chapter III. In addition to that, in view of the advent of new technologies, the authors are considering collecting ideas and information regarding new possible transportation services and developing necessary skills and measures, with specific target areas of this paper kept in mind.

\section{Acknowledgment}

This work was partly spported by Japan Society for the Promotion of Science, Grant-in-Aid for Scientific Research(C), No.15K00770 and FIVE, Non-Profit Organization.

\section{References}

[1] Banai-Kashanai, A.R., Toward a Synthetic Measure of Good Settlement Form" Environment and Planning B, 15, pp.399-412 (1988).

[2] N. Ohigashi, A. Tanaka and S. Watanabe, Traffic Awareness Investigation by Questionary Survey in Housing Complexs, Research bulletin of the Hiroshima Institute of Technology, Vol.43, pp.113-117 (2009), In Japanese.

[3] S. Matsumoto and N. Ohigashi, Examining an Effective Way to Support Vulnerable Road Users in Itsukaichi District, Hiroshima City, Information Engineering Express, Special issue on Business Management of Technology, Vol.2, No.3, pp.43-52 (2016).

[4] S. Matsumoto, N. Ohigashi, T. Hasuike, Developing a Transportation Support System for Vulnerable Road Users in Local Community, Proc. of 2016 5th IIAI International Congress on Advanced Applied Informatics, pp.797-800 (2016).

[5] D. Demailly, A. Novel, The sharing economy: make it sustainable. Studies, (03/14), 30 (2014). 
[6] J. Hamari, M. Sjoklint, A. Ukkonen, The sharing economy: Why people participate in collaborative consumption. Journal of the Association for Information Science and Technology, (2015).

[7] S. Matsumoto, N. Ohigashi and S. Abe, An equipment to support transportation of people and luggages in daily life, Japanese Patent Application No. 2015-160396 (2015), In Japanese.

[8] Y. Kawamoto, Car Corporative and Car Sharing Feasibility in the aged and Depopulated Village, Research report of Grant-in-Aid for Young Scientists (B), No.21760399 (20092010), In Japanese.

[9] T. Tsuboyama, K. Mizuno, H. Sasaki and S. Nishihara, Development of a simulation environment for dial-a-ride systems using swarm, Proceedings of the 75th National Convention of Information Processing Society of Japan, pp.129-131 (2013), In Japanese.

[10] K. Tsubouchi, H. Yamato and K. Hiekata, Development of the Simulator for the Ondemand Bus Introduction, The Japanese Society for Artificial Intelligence, Vol.25(3), pp.400-403 (2010), In Japanese.

[11] A. Kleiner, B. Nebel, V. Ziparo, A mechanism for dynamic ride sharing based on parallel auctions, Proc. of 22th International Joint Conference on Artificial Intelligence, pp.266-272 (2011).

[12] N. Agatz, A. Erera, M. Savelsbergh, X. Wang, Optimization for dynamic ride-sharing: A review, European Journal of Operational Research, Vol.223(2), pp.295-303 (2012).

[13] Nobuyuki Ohigashi and Shimpei Matsumoto, Research on the Possibility of Ridingtogether Traffic in Residential Estates, Bulletin of Hiroshima Institute of Technology, Research, Vol.49, pp.19-22 (2015), In Japanese.

[14] Y. Takamura, Ways of involvement of the government and the citizens towards solving the problems associated with the housing complexes in the suburb, Bulletin of the Faculty of Urban Management, Fukuyama City University, Vol.2, pp.33-50 (2013), In Japanese. 